\title{
THE GAUSS MAP AND A NONCOMPACT RIEMANN-ROCH FORMULA FOR CONSTRUCTIBLE SHEAVES ON SEMIABELIAN VARIETIES
}

\author{
J. Franecki, M. Kapranov
}

\begin{abstract}
For an irreducible subvariety $Z$ in an algebraic group $G$ we define an integer $\operatorname{gdeg}(Z) \geq 0$ as the degree, in a certain sense, of the Gauss map of $Z$. It can be regarded as a substitution for the intersection index of the conormal bundle to $Z$ with the zero section of $T^{*} G$, even though $G$ may not be compact. For $G$ a semiabelian variety (in particular, an algebraic torus $\left.\left(\mathbb{C}^{*}\right)^{n}\right)$, we prove a RiemannRoch type formula for constructible sheaves on $G$ which involves our substitutions for the intersection indicies. As a corollary, we get that a perverse sheaf on such a $G$ has nonnegative Euler characteristic, generalizing a theorem of Loeser-Sabbah.
\end{abstract}

\section{INTRODUCTION AND STATEMENT OF RESULTS}

Let $X$ be a smooth algebraic variety over $\mathbb{C}$ and $\mathfrak{F}$ be a constructible sheaf of $\mathbb{C}$-vector spaces on $X$. As in other situations, we have the Riemann-Roch problem: express $\chi(X, \mathfrak{F})=\sum_{i}(-1)^{i} \operatorname{dim} H^{i}(X, \mathfrak{F})$ in terms of some intrinsic geometric invariants of $X$ and $\mathfrak{F}$. One such invariant is the characteristic cycle $C C(\mathfrak{F})$ which is a formal $\mathbb{Z}$-linear combination $\sum_{\nu} n_{\nu}\left[\Lambda_{\nu}\right]$ of irreducible conic Lagrangian subvarieties $\Lambda_{\nu}$ in the cotangent bundle $T^{*} X$, see [14] for background. When $X$ is compact, the Riemann-Roch problem has a nice solution, namely [13]:

$$
\chi(X, \mathfrak{F})=(C C(\mathfrak{F}),[X])_{T^{*} X},
$$

where the right hand side is the intersection index, in $T^{*} X$, of $C C(\mathfrak{F})$ and the zero section $X \subset T^{*} X$. It can be calculated, for instance, by deforming $X$ to the graph of a $C^{\infty}$ 1-form so that the intersection becomes transverse, and then counting intersection points (with multiplicities and signs).

Both the definition of $C C(\mathfrak{F})$ and the formula (1.1) (for compact $X$ ) extend to the case when $\mathfrak{F}$ is a bounded constructible complex (i.e., a complex of sheaves with constructible cohomology).

When $X$ is not compact, $\chi(X, \mathfrak{F})$ still makes sense, but (1.1) is not applicable. We face, therefore, an interesting noncompact Riemann-Roch problem of finding $\chi(X, \mathfrak{F})$ in terms of invariants intrinsic to $X$ (in particular, not involving the choice of compactification).

The purpose of this paper is to exhibit such a "noncompact Riemann-Roch formula" in a particular class of situations. Namely, suppose that $X=G$ is an algebraic group with Lie algebra $\mathfrak{g}$. For $\gamma \in \mathfrak{g}^{*}$, let $\omega_{\gamma}$ be the corresponding leftinvariant 1-form on $G$, and $\Omega_{\gamma} \subset T^{*} G$ be its graph. The $\Omega_{\gamma}$ then form a natural family of deformations of $X$ and we can use them to make sense of the intersection 
index in (1.1) even when $G$ is not compact. More precisely, if $\Lambda \subset T^{*} G$ is an irreducible conic Lagrangian subvariety and $\gamma \in \mathfrak{g}^{*}$ is generic, then $\Lambda \cap \Omega_{\gamma}$ consists of finitely many transversal intersection points; their number will be denoted $\operatorname{gdeg}(\Lambda)$ and called the Gaussian degree of $\Lambda$. To explain the name, recall that $\Lambda$ has the form $T_{Z}^{*} X$ for an irreducible subvariety $Z \subset X$ (notation: $T_{Z}^{*} X$ will always mean the closure of the conormal bundle to the smooth locus $Z_{s m}$ of $Z$ ). Denoting $k=\operatorname{dim}(Z)$, we have the left Gauss map

$$
\Gamma_{Z}: Z \rightarrow G(k, \mathfrak{g}), z \mapsto z^{-1}\left(T_{z} Z\right) \subset T_{e} G=\mathfrak{g}
$$

which is a rational map, regular on $Z_{s m}$. The number $\operatorname{gdeg}(\Lambda)$ is the degree of $\Gamma_{Z}$ in an appropriate sense, see section 2 . For example, if $Z$ is a hypersurface, then the source and the target of $\Gamma_{Z}$ have the same dimension, and $\operatorname{gdeg}(\Lambda)$ is the degree of $\Gamma_{Z}$ in the usual sense.

Note that $\operatorname{gdeg}(\Lambda) \geq 0$ by construction. We now formulate the main result of this note. Recall [2] that a semiabelian variety is an algebraic group $G$ which is an extension

$$
1 \rightarrow T \rightarrow G \rightarrow A \rightarrow 1
$$

where $A$ is an abelian variety and $T \cong\left(\mathbb{C}^{*}\right)^{n}$ is an algebraic torus.

(1.3) Theorem. Let $G$ be a semiabelian variety, $\mathfrak{F}$ a bounded constructible complex on $G$ and $C C(\mathfrak{F})=\sum_{\nu} n_{\nu}\left[\Lambda_{\nu}\right]$. Then

$$
\chi(G, \mathfrak{F})=\sum_{\nu} n_{\nu} \operatorname{gdeg}\left(\Lambda_{\nu}\right) .
$$

(1.4) Corollary. If, in the situation of (1.3), $\mathfrak{F}$ is a perverse sheaf, then $\chi(G, \mathfrak{F}) \geq$ 0 .

Indeed, for $\mathfrak{F}$ a perverse sheaf, all $n_{\nu} \geq 0$. Here we use the conventions of [1] for the definition of (middle) perversity.

(1.5) Corollary. If $G$ is semiabelian and $Z \subset G$ is a smooth closed subvariety, then the number $(-1)^{\operatorname{dim}(Z)} \chi(Z, \mathbb{C})$ is nonnegative and coincides with $\operatorname{gdeg}(Z)$.

Indeed, $\mathbb{C}_{Z}[\operatorname{dim}(Z)]$ is perverse and its characteristic cycle is $\left[T_{Z}^{*} G\right]$ taken with multiplicity 1 .

Corollary 1.4 for $G=\left(\mathbb{C}^{*}\right)^{n}$ was proven by Loeser-Sabbah [12] and given a different proof (applicable to etale sheaves) by Gabber-Loeser [4]. In the case when $G$ is an abelian variety, Corollary 1.4 seems to be new, even though Theorem 1.3 in this case does not need a special proof, being a consequence of (1.1). Moreover, since the general proof of Theorem 1.3 given below is extremely simple and transparent, we believe that our approach exhibits the true reason behind the Loeser-Sabbah observation. Further, another result of [4] identifies irreducible perverse sheaves $\mathfrak{F}$ on $\left(\mathbb{C}^{*}\right)^{n}$ such that $\chi\left(\left(\mathbb{C}^{*}\right)^{n}, \mathfrak{F}\right)=1$, with complexes of solutions of hypergeometric systems (essentially, of the $A$-hypergeometric systems [5][11], see [6] for a comparison of the two points of view). On the other hand, in [12] the second author classified irreducible hypersurfaces in $\left(\mathbb{C}^{*}\right)^{n}$ for which the Gauss map has degree 1 and identified them with (reduced) $A$-discriminantal hypersurfaces. The 
latter describe the characteristic varieties of the $A$-hypergeometric system. Thus our approach explains the analogy between these two results.

Unfortunately, Theorem 1.3 cannot be straightforwardly generalized to more general algebraic groups. For example, if $G$ is nonabelian reductive, then it contains affine spaces $\mathbb{A}^{m}$ for $m$ ranging from 0 to the number of positive roots of $G$. The Euler characteristic of $\mathbb{A}^{m}$ being 1, Corollary 1.5 (and thus Theorem 1.3) cannot hold. The same applies when $G$, while commutative, contains $G_{a}$, the additive group. Nevertheless, we believe that there should exist generalizations of Theorem 1.3 which involve some particular classes of constructible sheaves and complexes.

We are grateful to V. Ginzburg for several useful remarks on the first version of the text. A part of the results of this paper were included in the thesis of the first author [3]. The second author was partially supported by the National Science Foundation.

\section{The Gauss map and the Gaussian Degree}

Let $G$ be a complex algebraic group, $\mathfrak{g}$ its Lie algebra and $G(k, \mathfrak{g})$ the Grassmannian of $k$-dimensional linear subspaces in $\mathfrak{g}$. If $Z \subset G$ is an irreducible $k$-dimensional subvariety, we have the rational map

$$
\Gamma_{Z}: Z \rightarrow G(k, \mathfrak{g})
$$

called the (left) Gauss map and defined as follows. For $x \in G$ let

$$
l_{x}: G \rightarrow G, l_{x}(y)=x y
$$

be the left translation by $x$. Then, for a smooth point $z \in Z$ the value $\Gamma_{Z}(z)$ is the image of

$$
d_{x} l_{x^{-1}}: T_{z} Z \rightarrow T_{e} G=\mathfrak{g} .
$$

We want to associate to $\Gamma_{Z}$ a nonnegative integer called its degree. To this end, consider first a more general situation.

Let $M$ be an irreducible $k$-dimensional variety, $V$ an $n$-dimensional vector space and $f: M \rightarrow G(k, V)$ a rational map. Replacing, if necessary, $M$ with its Zariski open subset, we can assume that $f$ is regular. Consider the flag variety $F(k, n-1, V)$ and its projections

$$
G(k, V) \stackrel{p}{\longleftarrow} F(k, n-1, V) \stackrel{q}{\longrightarrow} G(n-1, V)=P\left(V^{*}\right)
$$

Let $\widetilde{M}=G(k, V) \times{ }_{M} F(k, n-1, V)$ be the fiber product with respect to $f$ and $p$. Since $p$ is a smooth map with $(n-k-1)$-dimensional fibers, $\widetilde{M}$ is an irreducible variety of dimension $n-1$. The map $q$ induces a regular map $q^{\prime}: \widetilde{M} \rightarrow \mathbb{P}\left(V^{*}\right)$ whose source and target have the same dimension. We define $\operatorname{deg}(f)$ to be the degree of the map $q^{\prime}$. The following is then clear.

(2.1) Proposition. If $W \subset V$ is a generic hyperplane, then $\operatorname{deg}(f)$ is equal to the number of $x \in M$ such that $f(x) \in G(k, W) \subset G(k, V)$. For any such $x$ the map $f$ is locally (in the analytic or etale cohomology) an embedding near $x$ and the intersection of $f(M)$ and $G(k, W)$ is transversal at $x$.

When $k=n-1$, we have $\widetilde{M}=M$ and $\operatorname{deg}(f)$ is the degree of $f$ in the usual sense. 
We now specialize to the case $M=Z, V=\mathfrak{g}, n=\operatorname{dim}(G)$ and $f=\Gamma_{Z}$. The number $\operatorname{deg}\left(\Gamma_{Z}\right)$ will be denoted by $\operatorname{gdeg}(Z)$ and called the Gaussian degree of $Z$.

Let $\Lambda=T_{Z}^{*} G$ be the conic Lagrangian variety associated to $Z$. We will write $\operatorname{gdeg}(\Lambda)$ for $\operatorname{gdeg}(Z)$. As in Section 1 , for $\gamma \in \mathfrak{g}^{*}$ let $\Omega_{\gamma} \subset T^{*} X$ be the graph of the left-invariant1-form $\omega_{\gamma}$ on $G$ associated to $\gamma$. Proposition 2.1 implies easily:

(2.2) Proposition. Let $\gamma \in \mathfrak{g}^{*}$ be a generic linear functional. Then $\Lambda \cap \Omega_{\gamma}$ consists of finitely many points which are smooth on $\Lambda$ and in which the intersection is transverse. The number of these points is equal to gdeg $(\Lambda)$.

\section{Characteristic Cycle of an Open embedding}

A non-intrinsic way to find $\chi(U, \mathfrak{F})$ where $U$ is a noncompact manifold, is to apply (1.1) to $R j_{*} \mathfrak{F}$ where $j: U \hookrightarrow X$ is a smooth compactification. We will indeed use this approach in the proof of Theorem 1.3, so we recall the (now well known) proceedure of finding $C C\left(R j_{*} \mathfrak{F}\right)$ from $C C(\mathfrak{F})$, see [7] [17].

Let $X$ be a not necessarily compact smooth variety, $f \in \mathbb{C}[X]$ a regular function, $U \subset X$ the open set $\{f \neq 0\}$, and $j: U \hookrightarrow X$ the embedding. Let $\Lambda \subset T^{*} U$ be an irreducible conic Lagrangian variety. For $s \in \mathbb{C}^{*}$ let

$$
\Lambda_{s}^{\#}=\Lambda+s d \log f=\{(\xi+s(d \log f)(x), x) \mid(x, \xi) \in \Lambda\}
$$

This is a closed (no longer conic) Lagrangian subvariety in $T^{*} X$. The total space of the family of $\Lambda_{s}^{\#}$ is a subvariety $\Lambda^{\#} \subset T^{*} X \times \mathbb{C}^{*}$. The limit $\lim _{s \rightarrow 0} \Lambda_{s}^{\#}$ (also called the specialization of $\Lambda^{\#}$ in [7]) is an effective Lagrangian cycle in $T^{*} X$ defined as follows. We first take the closure $\overline{\Lambda^{\#}}$ in $T^{*} X \times \mathbb{C}$ and then form the schemetheoretic intersection $\overline{\Lambda^{\#}} \cap\left(T^{*} X \times\{0\}\right)$. The cycle $\lim _{s \rightarrow 0} \Lambda_{s}^{\#}$ is obtained by taking the irreducible components of this intersection with the multiplicities given by the scheme structure.

We extend this construction by $\mathbb{Z}$-linearity to conic Lagrangian cycles in $T^{*} U$. Thus, if $\Sigma$ is such a cycle, we have the family of non-conic cycles $\Sigma_{s}^{\#}, s \in \mathbb{C}^{*}$ and the conic cycle $\lim _{s \rightarrow 0} \Sigma_{s}^{\#}$ in $T^{*} X$. Now, the fact we need is as follows.

(3.2) Theorem. If $\mathfrak{F}$ is a bounded constructible complex on $U$, then

$$
C C\left(R j_{*} \mathfrak{F}\right)=\lim _{s \rightarrow 0} C C(\mathfrak{F})_{s}^{\#}
$$

This statement can be obtained from Theorem 3.2 of [7] by applying the RiemannHilbert correspondence, or from Theorem 3.1 of [17] which is applicable to the more general case of $\mathbb{R}$-constructible sheaves. (To be precise, the concepts of the characteristic cycle used in [7-9] and [13][14][17] refer to different contexts: holonomic D-modules vs. constructible complexes. The compatibility of these two definitions of the characteristic cycle inder the Riemann-Hilbert correspondence follows from the results of [7].)

Consider now a nominally more general situation (cf. [9], Appendix A): let $X$ be as before but suppose that we have $n$ regular functions $f_{1}, \ldots, f_{n} \in \mathbb{C}[X]$. Let $U$ be the intersection of the $n$ open sets $\left\{f_{i} \neq 0\right\}$ and $j: U \hookrightarrow X$ be the embedding. Of course, this situation can be analyzed by applying Theorem 3.2 to $f=f_{1} \ldots f_{n}$, but it will be convenient for us to have a more flexible formulation. 
For a point $s=\left(s_{1}, \ldots, s_{n}\right) \in\left(\mathbb{C}^{*}\right)^{n}$ and a conic Lagrangian variety $\Lambda \subset T^{*} U$ we form, similarly to (3.1), a non-conic Lagrangian variety

$$
\Lambda_{s}^{\#}=\Lambda+\sum_{i=1}^{n} s_{i} d \log f_{i} \subset T^{*} X .
$$

The total space of this family lies in $T^{*} X \times\left(\mathbb{C}^{*}\right)^{n}$. Taking the closure in $T^{*} X \times \mathbb{C}^{n}$ and then intersecting with $T^{*} X \times\{(0, \ldots, 0)\}$ defines, similarly to the above, a conic Lagrangian cycle $\lim _{s \rightarrow(0, \ldots, 0)} \Lambda_{s}^{\#}$. Of course, this "limit" could be taken along any curve in $\mathbb{C}^{n}$ passing through 0 and generically lying in $\left(\mathbb{C}^{*}\right)^{n}$. As before, we extend this construction by linearity to conic Lagrangian cycles in $T^{*} U$. The next statement follows by iterated application of Theorem 3.2.

(3.4) Theorem. If $\mathfrak{F}$ is a bounded constructible complex on $U$, then

$$
C C\left(R j_{*} \mathfrak{F}\right)=\lim _{s \rightarrow(0, \ldots, 0)} C C(\mathfrak{F})_{s}^{\#}, s=\left(s_{1}, \ldots, s_{n}\right) \in\left(\mathbb{C}^{*}\right)^{n} .
$$

Taking the limit along different curves approaching $(0, \ldots, 0)$ corresponds, roughly, to different choices of our equation for the reducible hypersurface $X \backslash U$. For example, restricting to the curve with parametric equation $s_{i}=t^{m_{i}}, m_{i}>0$ corresponds to taking $\prod_{i} f_{i}^{m_{i}}$ as an equation.

We now need a slight globalization of Theorem 3.4. First of all, let $(L, \nabla)$ be a line bundle on $X$ with an algebraic flat connection. If $f$ is a regular section of $L$, then $f^{-1} \nabla f$ is a scalar 1 -form regular over the open set $\{f \neq 0\}$. We denote this form $\nabla \log f$.

Suppose now that we have $n$ line bundles with flat connections $\left(L_{i}, \nabla_{i}\right)$ on $X$, $i=1, \ldots, n$. Suppose $f_{i} \in \Gamma\left(X, L_{i}\right), i=1, \ldots, n$ and $U \subset X$ is the intersection of the open sets $\left\{f_{i} \neq 0\right\}$. As before, let $j: U \hookrightarrow X$ be the embedding.

(3.5) Theorem. For a bounded constructible complex $\mathfrak{F}$ on $U$ we have

$$
C C\left(R j_{*} \mathfrak{F}\right)=\lim _{s \rightarrow(0, \ldots, 0)}\left(C C(\mathfrak{F})+\sum_{i=1}^{n} s_{i} \nabla_{i} \log f_{i}\right) .
$$

Proof: As before, it is enough to consider the case $n=1$, as the general case can be obtained by iteration, as in [9], Appendix A. The statement for $n=1$ is a consequence of Theorem 6.3 of [8] which deals with the more general case of the zero locus of a section $f$ of an arbitrary line bundle $L$, not necessarily with connection. The recipe in this case is to consider the "twisted cotangent bundles" $\left(T^{*} X\right)^{(s)}, s \in \mathbb{C}$, defined as the symplectic quotients of $T^{*} L$ by the hamiltonian action of $\mathbb{C}^{*}$ induced by dilations of $L$. Now, if $L$ is equipped with a flat connection $\nabla$, then all the $\left(T^{*} X\right)^{(s)}$ become identified with $T^{*} X$ and the formulation of [8], Theorem 6.3 reduces to our statement.

\section{Proof of Theorem 1.3 for $G=\left(\mathbb{C}^{*}\right)^{n}$}

We first consider the case when $G=\left(\mathbb{C}^{*}\right)^{n}$ is an algebraic torus. Let $z_{1}, \ldots, z_{n}$, $z_{i} \neq 0$, be the standard coordinates in $\left(\mathbb{C}^{*}\right)^{n}$. We compactify $G$ by the projective space $\mathbb{P}^{n}$ with homogeneous coordinates $\left(t_{0}: \cdots: t_{n}\right)$ by

$$
j:\left(\mathbb{C}^{*}\right)^{n} \hookrightarrow \mathbb{P}^{n},\left(z_{1}, \ldots, z_{n}\right) \mapsto\left(1: z_{1}: \cdots: z_{n}\right)
$$


For $\nu=0, \ldots, n$ let $A_{\nu}^{n} \subset \mathbb{P}^{n}$ be the affine chart given by $t_{\nu} \neq 0$. This is an affine space with coordinates $z_{i}^{(\nu)}, i \in\{0, \ldots, n\} \backslash\{\nu\}$ given by $z_{i}^{(\nu)}=\frac{t_{i}}{t_{\nu}}$. Denote by

$$
\left(\mathbb{C}^{*}\right)^{n} \stackrel{j_{\nu}}{\longleftrightarrow} A_{\nu}^{n} \stackrel{k_{\nu}}{\longleftrightarrow} \mathbb{P}^{n}
$$

the embeddings. For $\nu=0$ we have $z_{i}^{(0)}=z_{i}$.

We now apply Theorem 3.4 to $U=\left(\mathbb{C}^{*}\right)^{n}, X=A_{0}^{n}, f_{i}=z_{i}$, and our constructible complex $\mathfrak{F}$. The recipe of the theorem requires us to introduce the family of 1 -forms

$$
\omega_{s}=\sum_{i=1}^{n} s_{i} d \log z_{i}, s=\left(s_{1}, \ldots, s_{n}\right) \in\left(\mathbb{C}^{*}\right)^{n} .
$$

These forms are precisely the invariant 1 -forms on $G=\left(\mathbb{C}^{*}\right)^{n}$. We can view $s$ as an element of $\mathfrak{g}^{*}$, where $\mathfrak{g}=\mathbb{C}^{n}$ is the Lie algebra of $G$. Theorem 3.4 then gives us:

$$
C C\left(R j_{0 * \mathfrak{F}} \mathfrak{F}\right)=\left.C C\left(R j_{*} \mathfrak{F}\right)\right|_{A_{0}^{n}}=\lim _{s \rightarrow 0}\left(C C(\mathfrak{F})+\omega_{s}\right),
$$

the limit being taken in $T^{*} A_{0}^{n}$.

Next, we apply Theorem 3.4 to $U=\left(\mathbb{C}^{*}\right)^{n}$ and $X=A_{\nu}^{n}$ with arbitrary $\nu \in$ $\{0, \ldots, n\}$. Then we should take $f_{i}=z_{i}^{(\nu)}, i \in\{0, \ldots, n\} \backslash\{\nu\}$ and consider the 1 -forms

$$
\omega_{s^{\prime}}^{(\nu)}=\sum_{i \neq \nu} s_{i}^{\prime} d \log z_{i}^{(\nu)}, s^{\prime} \in\left(\mathbb{C}^{*}\right)^{\{0, \ldots, n\} \backslash\{\nu\}} .
$$

Now, each $z_{i}^{(\nu)}$ is a Laurent monomial in the $z_{1}, \ldots, z_{n}$, so $d \log z_{i}^{(\nu)}$ is an (integer) linear combination of $d \log z_{1}, \ldots, d \log z_{n}$. Therefore, $\omega_{s^{\prime}}^{(\nu)}=\omega_{s}$ where $s=\phi_{\nu}\left(s^{\prime}\right)$ is an image of $s$ under a linear transformation $\phi_{\nu}: \mathbb{C}\{0, \ldots, n\} \backslash\{\nu\} \rightarrow \mathbb{C}^{n}$. It is clear that $\phi_{\nu}$ is invertible; in particular, $s \rightarrow 0$ iff $s^{\prime} \rightarrow 0$. This means that the answers

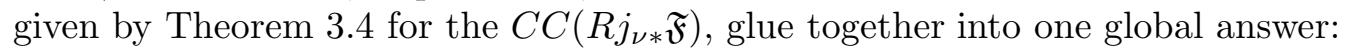

$$
C C\left(R j_{*} \mathfrak{F}\right)=\lim _{\substack{s \rightarrow 0 \\ s \in E}}\left(C C(\mathfrak{F})+\omega_{s}\right) .
$$

Here the limit is taken in $T^{*} \mathbb{P}^{n}$ and $s$ runs over the set

$$
E=\left\{\left(s_{1}, \ldots, s_{n}\right) \in\left(\mathbb{C}^{*}\right)^{n}: s_{i} \neq s_{j}, i \neq j\right\} .
$$

(The condition $s \in E$ is equivalent to $\phi_{\nu}^{-1}(s) \in\left(\mathbb{C}^{*}\right)^{\{0, \ldots, n\} \backslash\{\nu\}}$ for any $\nu$.)

Now, Theorem 1.3 for $G=\left(\mathbb{C}^{*}\right)^{n}$ would follow from (4.2) and the next lemma.

(4.3) Lemma. Let $\Lambda \subset T^{*}\left(\mathbb{C}^{*}\right)^{n}$ be an irreducible conic Lagrangian variety. Then

$$
\left.\lim _{\substack{s \rightarrow 0 \\ s \in E}}\left(\Lambda+\omega_{s}\right),\left[\mathbb{P}^{n}\right]\right)_{T * \mathbb{P}^{n}}=\operatorname{gdeg}(\Lambda) .
$$

Proof: Let $\Omega_{s} \subset T^{*}\left(\mathbb{C}^{*}\right)^{n}$ be the graph of $\omega_{s}$. If $s \in E$, then $\Omega_{s}$ is closed in $T^{*} \mathbb{P}^{n}$ as well. By translation, intersecting $\Lambda+\omega_{s}$ with $\left[\mathbb{P}^{n}\right]$ is equivalent to intersecting $\Lambda$ with $\Omega_{-s}$. By Proposition 2.2, there exists a Zariski open, nonempty set $F \subset \mathbb{C}^{n}$ such that for $s^{(0)} \in F$ the intersection $\Lambda \cap \Omega_{-s^{(0)}}$ consists of $\operatorname{gdeg}(\Lambda)$ smooth transverse points. Since $E$ is also Zariski open, $F \cap E$ meets any polydisk

$$
P_{\varepsilon}=\left\{\left(z_{1}, \ldots, z_{n}\right) \in\left(\mathbb{C}^{*}\right)^{n}: 0<\left|z_{i}\right|<\varepsilon\right\}
$$

in an open dense set. By the "continuity of intersection" it follows that for $|\varepsilon| \ll 1$ and $s^{(0)} \in P_{\varepsilon} \cap F \cap E$

$$
\left.\left(\lim _{\substack{s \rightarrow 0 \\ s \in E}} \Lambda+\omega_{s}\right),\left[\mathbb{P}^{n}\right]\right)_{T^{*} \mathbb{P}^{n}}=\left|\Omega_{-s(0)} \cap \Lambda\right|=\operatorname{gdeg}(\Lambda)
$$

and this completes the proof. 


\section{Proof of ThEOREM 1.3 IN GENERAL}

Let $A$ be an abelian variety. We recall some elementary properties of line bundles on $A$, see [10] [16]. If $L$ is such a bundle, by $L_{a}, a \in A$ we denote its fiber at $a$. By $L^{0}$ we denote the total space of $L$ with the zero section deleted, so $L^{0}$ is a principal $\mathbb{C}^{*}$-bundle on $A$. As for any base variety, the correspondence $L \mapsto L^{0}$ is an equivalence between the category of line bundles and isomorphisms and the category of principal $\mathbb{C}^{*}$-bundles.

Assume that $L$ has degree 0 . Then the theorem of the square [16] provides identifications

$$
L_{0} \cong \mathbb{C}, L_{a} \otimes L_{b} \simeq L_{a+b}
$$

which make $L^{0}$ into a group, namely a semiabelian variety fitting into an extension

$$
0 \rightarrow \mathbb{C}^{*} \rightarrow L^{0} \stackrel{p}{\longrightarrow} A \rightarrow 0 .
$$

Next, any bundle $L$ of degree 0 has a flat connection. All such connections form an affine space $\operatorname{Conn}(L)$ over the vector space $H^{0}\left(A, \Omega^{1}\right)=\mathfrak{a}^{*}$. Here $\mathfrak{a}$ is the Lie algebra of $A$. Let $\mathfrak{L}$ be the Lie algebra of the group $L^{0}$, so that we have the exact sequence

$$
0 \rightarrow \mathfrak{a}^{*} \rightarrow \mathfrak{L}^{*} \stackrel{\pi}{\longrightarrow} \mathbb{C} \rightarrow 0
$$

Denote by $\widetilde{L}=p^{*} L$ the pullback of $L$ to $L^{0}$. For any $\nabla \in \operatorname{Conn}(L)$ let $\widetilde{\nabla}$ be its pullback to a connection in $\widetilde{L}$. Denote also by $f$ the tautological section of $\widetilde{L}$ over $L^{0}$ (given by the identity map).

(5.3) Proposition. The 1-forms $\widetilde{\nabla} \log f, \nabla \in \operatorname{Conn}(L)$, are invariant (with respect to the group structure on $\left.L^{0}\right)$. Their images in $\mathfrak{L}^{*}$ under the evaluation at 0 form the subspace $\pi^{-1}(1)$, where $\pi$ is as in (5.2).

Proof: Let us first show the invariance. Note that $\widetilde{\nabla} \log f$ is just the Lie algebravalued 1 -form on the total space of the principal $\mathbb{C}^{*}$-bundle $L^{0}$, describing the connection $\nabla$ in $L^{0}$ in the standard approach of differential geometry. So its invariance follows from the fact that the line bundle $(L, \nabla)$ (or, what is equivalent, the $\mathbb{C}^{*}$-bundle $\left.\left(L^{0}, \nabla\right)\right)$ satisfies the theorem on the square as a bundle with connection. More precisely, if $m, q_{1}, q_{2}: A \times A \rightarrow A$ are the group structure and the two projections, then the isomorphism

$$
\mu: q_{1}^{*} L \otimes q_{2}^{*} L \rightarrow m^{*} L
$$

given by the theorem on the square is an isomorphism of bundles with connection. In particular, the induced isomorphism $\mu_{a}: L_{a} \otimes L \rightarrow l_{a}^{*} L$ is an isomorphism of bundles with connection on $A$. But the translation $l_{(a, \lambda)}, \lambda \in L_{a} \backslash\{0\}$ on $L^{0}$ is just given by $\mu_{a}(\lambda \otimes-)$. This shows the invariance. As for the second assertion of Proposition 5.3, it is again obvious from the interpretation of $\widetilde{\lambda} \log f$ as the Lie algebra-valued 1-form describing the connection and the identification $L_{0}^{0}=\mathbb{C}^{*}$.

(5.4) Corollary. The 1-forms $s \widetilde{\nabla} \log f, s \in \mathbb{C}^{*}, \nabla \in \operatorname{Conn}(L)$, form a nonempty Zariski open set in the space of all invariant 1-forms on $L^{0}$.

Consider now several line bundles of degree 0 , say $L_{1}, \ldots, L_{n}$, on $A$, and let

$$
0 \rightarrow\left(\mathbb{C}^{*}\right)^{n} \rightarrow G=L_{1}^{0} \times_{A} \ldots \times_{A} L_{n}^{0} \stackrel{p}{\longrightarrow} A \rightarrow 0
$$


be the associated semiabelian variety.

We denote $\widetilde{L}_{i}=p^{*} L_{i}$ and let $f_{i} \in H^{0}\left(G, \widetilde{L}_{i}\right)$ be the tautological section. As before, for $\nabla_{i} \in \operatorname{Conn}\left(L_{i}\right)$ we denote by $\widetilde{\nabla}_{i}$ its pullback to $\widetilde{L}_{i}$. Corollary 5.4 implies easily:

(5.6) Proposition. Suppose $n \geq 0$. Then, the 1 -forms $\sum_{i=1}^{n} s_{i} \widetilde{\nabla}_{i} \log f_{i}$ for $s_{1}, \ldots, s_{n} \in$ $\mathbb{C}^{*}, \nabla_{i} \in \operatorname{Conn}\left(L_{i}\right)$, form a Zariski open dense set in the space of all invariant 1forms on $G$.

We now turn to the proof of Theorem 1.3. Our approach is similar to [18], $\S 2$. First of all, it is known that any semiabelian variety has the form (5.5) which we assume. Next, we assume $n>0$ since for $n=0$ the group $G=A$ is compact and the theorem follows from (1.1) and from Proposition 2.2. We set $L_{0}=\mathcal{O}_{A}$ and compactify $G$ by embedding it into the relative projectivization

$$
j: G \hookrightarrow \mathbb{P}:=\mathbb{P}\left(L_{0} \oplus \ldots \oplus L_{n}\right) \stackrel{\rho}{\rightarrow} A .
$$

We can think of $\mathbb{P}$ as having homogeneous coordinates $\left(t_{0}: \ldots ; t_{n}\right)$ with $t_{i}$ being not a function any more but rather a section of $\rho^{*} L_{i}$. Note that $\rho^{*} L_{i}$ has a flat connection induced from $\nabla_{i}$, whose restriction to $G$ is $\widetilde{\nabla}_{i}$. The variety $\mathbb{P}$ is the union of the relative affine charts $A_{\nu}=\bigoplus_{i \neq \nu} L_{i}$. Inside $A_{0}$, the complement of $G$ is given by the condition that one of the tautological sections (still denoted by $f_{i}$ ) of the pullback of $L_{i}$, vanishes.

Take generic $\nabla_{i} \in \operatorname{Conn}\left(L_{i}\right)$ so that for a Zariski open, dense set of $s \in\left(\mathbb{C}^{*}\right)^{n}$ the 1-form $\omega_{s}=\sum s_{i} \widetilde{\nabla}_{i} \log f_{i}$ satisfies Proposition 2.2. Then, we mimic the arguments of Section 4 but in the relative situation, using Theorem 3.5. We find that

$$
C C\left(R j_{*} \mathfrak{F}\right)=\lim _{\substack{s \rightarrow 0 \\ s \in E}}\left(C C(\mathfrak{F})+\sum_{i} s_{i} \widetilde{\nabla}_{i} \log f_{i}\right),
$$

the limit being taken in $T^{*} \mathbb{P}$. After this, the proof is identical to the argument at the end of Section 4, and the theorem is proven.

\section{REFERENCES}

[1] A.A. Belinson, J.Bernstein, P. Deligne, Faiceaux Pervers, Asterisque 100 (1982), pp. 5-171.

[2] C.Chai, G. Faltings, Degeneration of Abelian Varieties, Springer-Verlag, 1990.

[3] J. Franecki, The Gauss Map and Euler Characteristic on Algebraic Groups, Thesis, Northwestern University, 1998.

[4] O. Gabber, F. Loeser, Faisceaux pervers l-adiques sur un tore, Duke Mathematical Journal 83 (1996), no. 3, pp. 501-606.

[5] I.M. Gelfand, M.M. Kapranov, A.V. Zelevinsky, Generalized Euler integrals and A-hypergeometric functions, Adv. in Math. 84 (1990), no. 2, pp. 255-271.

[6] I.M. Gelfand, M.M. Kapranov, A.V. Zelevinsky, Hypergeometric functions, toric varieties and newton polyhedra, in: "Special Functions" ( ICM-90 Sattelite Conference Proceedings, M. Kashiwara, T. Miwa, Eds.) p. 104-121, Springer-Verlag, Berlin, 1991.

[7] V. Ginsburg, Characteristic varieties and vanishing cycles, Invent. Math. 84 (1986), pp. $327-402$.

[8] V. Ginsburg, G-modules, Springer's representations and bivariant Chern classes, Adv. in Math. 61 (1986), 1-48. 
[9] V. Ginzburg, Admissible modules on a symmetric space, Astérisque 173-74 p.199-255, Soc. Math. France, 1989.

[10] P. Griffiths, J. Harris, Principles of Algebraic Geometry, John Wiley \& Sons, Inc., 1978.

[11] J. Horn, Über die Konvergenz hypergeometrischer Reihen zweier und dreier Veränderlichen, Math. Ann. 34 (1889), pp. 544-600.

[12] M.M. Kapranov, A characterization of A-discriminantal hypersurfaces in terms of the logarithmic Gauss map, Math. Ann. 290 (1991), pp. 277-285.

[13] M. Kashiwara, Index theorem for constructible sheaves, Asterisque 130 (1985), pp. 193 209 .

[14] M. Kashiwara, P. Schapira, Sheaves on Manifolds, Springer-Verlag, 1990.

[15] F. Loeser, C. Sabbah, Caractérisation des D-modules hypergéometriques irreductibles sur le tore, Comptes Rendus Serie I. (Mathématiques), 312 (1991), no. 10, pp. 735 - 738, continued in 315 (1992), no. 12, pp. 1263 - 1264.

[16] D. Mumford, Abelian Varieties, Oxford University Press, 1970.

[17] W. Schmid, K. Vilonen, Characters, characteristic cycles, and nilpotent orbits, in: Geometry, Topology, and Physics (S.-T. Yau, Ed.), pp. 329-340, International Press, 1995.

[18] P. Vojta, Integer points on subvarieties of semiabelian varieties I, Invent. Math. 126 (1996), 133-181.

J.F.: Department of Mathematics, Loyola University, 6525 N. Sheridan Rd. Chicago IL 60620,

M.K.: Department of Mathematics, Northwestern University, Evanston IL 60208

E-mail address: franecki@math.luc.edu, kapranov@math.nwu.edu 